\title{
NOVEL 24-MEMBERED OCTANUCLEAR MANGANESE(III) METALLACROWN
}

\author{
Fangfang Dang ${ }^{1 *}$, Dangyang Zhao ${ }^{2}$, Hui Guo ${ }^{3}$ and KeWei Lei $^{4}$
}

${ }^{1}$ School of Science, Xi'an University of Architecture and Technology, Xi'an, China; ${ }^{2}$ Luohe Environmental Sanitation Management Agency, Luohe, China; ${ }^{3}$ Henan Yinge Industrial Investment Co. Ltd., Luohe, China; ${ }^{4}$ State Key Laboratory Base of Novel Functional Materials and Preparation Science, Institute of Solid Materials Chemistry, Faculty of Materials Science and Chemical Engineering, Ningbo University, Ningbo, China

(Received September 9, 2013; revised September 3, 2014)

ABSTRACT. A novel octanuclear manganese metalladiazamacrocycle (1) was synthesized employing a new pentadentate ligand $\mathrm{N}$-methacryl-5-methylsalicylhydrazide $\left(\mathrm{H}_{3}\right.$ mamshz) by supramolecular self assembly. The backbone of this metal-organic assembly is a repeating unit of $\mathrm{Mn}-\mathrm{N}-\mathrm{N}-\mathrm{Mn}$ linkage that extends to complete a 24-membered cyclic structure involving 8 manganese(III) centers. Successive manganese centers are in an octanuclear cyclic structure. The temperature-dependent magnetic properties show a typical weakly coupled antiferromagnetic behaviour.

KEY WORDS: Manganese, Octanuclear, Metalladiazamacrocycle, Pentadentate ligand, Antiferromagnetic behavior

\section{INTRODUCTION}

Metallamacrocycles have become important in recent years because of their interesting molecular architecture [1], multinuclear structures [2] and magnetic properties [3]. They have also been used as building blocks for the construction of two- or three-dimensional network structures [4]. A variety of metallamacrocycles and cages were found to form interesting hostguest systems with different metal ions of varying coordination and symmetry [5]. Metal ions such as $\mathrm{Ga}, \mathrm{Co}, \mathrm{Fe}$ and $\mathrm{Mn}$ that can easily form stable octahedral coordination, are found to yield hexanuclear metallamacrocycles with trianionic pentadentate ligands [6]. However manganese and iron have received special attention because of their ease of formation of metallamacrocycles and interesting magnetic properties [7]. Controlling the size and nuclearity of metallamacrocycles and their properties has quite recently become of interest. Although there have been reports of metallamacrocycles and metallacrowns of high nuclearity containing iron and manganese, formation of stable cyclic high nuclearity structures has been an uphill task.

We report here a novel 24-membered octanuclear manganese metalladiazamacrocycle, $\left[\mathrm{Mn}_{8}(\text { mamshz })_{8}(\mathrm{DMA})_{8}\right]\left[\mathrm{Mn}_{8}(\text { mamshz })_{8}(\mathrm{DMA})_{6}\left(\mathrm{H}_{2} \mathrm{O}\right)_{2}\right] \cdot 2 \mathrm{DMA} \cdot 4 \mathrm{H}_{2} \mathrm{O}$ (1), a cyclic structure derived from employing a trianionic pentadentate ligand $\mathrm{N}$-methacryl-5-methylsalicylhydrazide $\left(\mathrm{H}_{3}\right.$ mamshz) (Scheme 1).
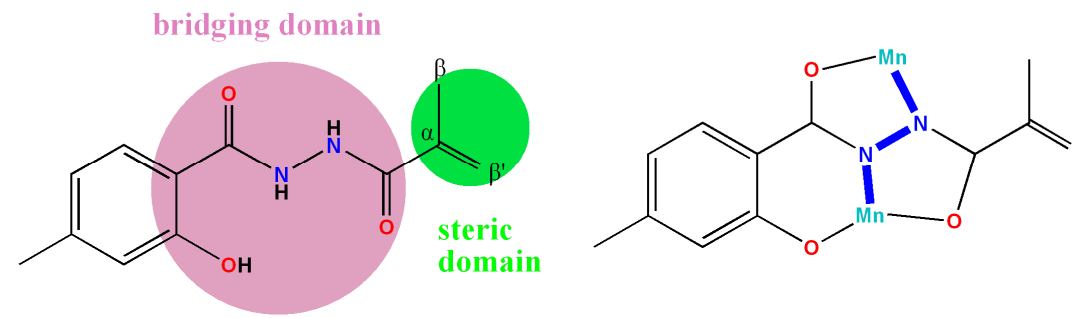

Scheme 1. Ligand and basic binding sites of compound $\mathbf{1}$.

*Corresponding author. E-mail: dff516@163.com 


\section{EXPERIMENTAL}

\section{Materials and physical measurements}

All chemicals used were of analytical grade and were used in this experiment without further purification.

Carbon, nitrogen and hydrogen analyses were determined using a Vario EL elemental analyzer. IR spectra were recorded on a Nicolet Avatar 360 FT-IR instrument using $\mathrm{KBr}$ discs in the $400-4000 \mathrm{~cm}^{-1}$ region. ${ }^{1} \mathrm{H}$ NMR spectra were measured on a FT-400A spectrometer in dmso- $d_{6}$ solution, with TMS as internal standard. The intensity data were collected at $296 \mathrm{~K}$ on a Bruker Smart APEX II diffractometer with graphite-monochromatized Mo K $\alpha$ radiation $(\lambda=$ $0.71073 \AA$ ). The structure was solved by direct methods and refined by full matrix least-squares techniques on $F^{2}$ with all non-hydrogen atoms treated anisotropically. All calculations were performed with the SHELXTL program package. Non-hydrogen atoms with geometrical rigidity were refined anisotropically, but non-hydrogen atoms with geometrical flexibility were refined isotropically; hydrogen atoms attached to the non-disordered part were assigned isotropic displacement coefficients $U(\mathrm{H})=1.2 U(\mathrm{C})$ or $1.5 U\left(\mathrm{C}_{\text {methyl }}\right)$, and their coordinates were allowed to ride on their respective atoms. Temperature-dependent magnetic susceptibility measurements were carried out on powdered samples between 2 and $300 \mathrm{~K}$ using a Quantum Design MPMS7XL SQUID magnetometer. Field-cooled magnetization data were collected at $H=1000$ Oe. The diamagnetic correction for complex was calculated using Pascal's constants.

Preparation of $\mathrm{H}_{3}$ mamshz

To a $35 \mathrm{~mL}$ of chloroform solution at $0{ }^{\circ} \mathrm{C}$, containing $3.09 \mathrm{~mL}(22 \mathrm{mmol})$ of triethylamine and $1.72 \mathrm{~g}(20 \mathrm{mmol})$ of methacrylic acid was added $2.49 \mathrm{~mL}(20 \mathrm{mmol})$ of trimethylacetyl chloride with stirring. The solution was then brought slowly to room temperature. After stirring for 30 min $2.32 \mathrm{~g}(20 \mathrm{mmol})$ of 2-hydroxy-4-methylbenzohydrazide was added to it and stirring was continued for another $40 \mathrm{~min}$. It is then layered with $20 \mathrm{~mL}$ of hexane; a white suspension appears which was then left aside for complete precipitation. The product $\left(\mathrm{H}_{3} \mathrm{mamshz}\right)$ obtained was filtered, washed successively with a 1:1 mixture of chloroform and hexane, and then with ether. The compound was recrystallised from ethanol to yield $2.80 \mathrm{~g}(60 \%)$. Elemental data for $\mathrm{C}_{12} \mathrm{H}_{14} \mathrm{~N}_{2} \mathrm{O}_{3}$ calc: $\mathrm{C} 61.53, \mathrm{H} 6.02, \mathrm{~N} 11.96 \%$; found: C 61.64, H 6.18, N 11.82\%. ${ }^{1} \mathrm{H}$ NMR (400 $\left.\mathrm{MHz}, \mathrm{dmso}-d_{6}, \mathrm{ppm}\right): \delta 12.00(1 \mathrm{H}, \mathrm{s}, \mathrm{ArOH}) ; 10.47(1 \mathrm{H}, \mathrm{s}, \operatorname{ArCONH}) ; 10.12(1 \mathrm{H}, \mathrm{s}$, MeCONH); 7.80-7.78 (1H, d, J = 8 Hz, o-ArH); 6.78-6.75 (1H, t, J = 8.8 Hz, m-ArH); 5.82 $\left(1 \mathrm{H}, \mathrm{s},-\mathrm{CO}-\mathrm{C}-\mathrm{CH}_{2}-\right) ; 5.51\left(1 \mathrm{H}, \mathrm{s},-\mathrm{CO}-\mathrm{C}-\mathrm{CH}_{2}-\right) ; 2.29\left(3 \mathrm{H}, \mathrm{s}, \mathrm{ArCH}_{3}\right) ; 1.92\left(3 \mathrm{H}, \mathrm{s}, \mathrm{COCH}_{3}\right)$. IR $\left(\mathrm{KBr}\right.$ pellet, $\left.\mathrm{cm}^{-1}\right)$ : 3324(s), 3103(w), 3066(w), 3016(w), 2980(w), 2931(w), 2879(w), 2738(m), 2717(w), 2681(w), 2623(w), 2603(w), 2526(w), 2495(w), 1662(s), 1647(m), 1614(s), 1545(s), 1514(s), 1481(s), 1423(m), 1375(m), 1315(s), 1286(s), 1250(s), 1209(s), 1174(s), 1145(s), 1119(s), 1038(m), 1014(s), 957(s), 924(s), 881(s), 835(s), 827(s), 816(m), 769(s), 737(m), 704(m), 673(w), 596(s), 559(s), 509(w), 455(w), 436(m), 411(w).

Preparation of compound 1. The dark brown block crystals of $\left[\mathrm{Mn}_{8}(\text { mamshz })_{8}(\mathrm{DMA})_{8}\right]\left[\mathrm{Mn}_{8}(\mathrm{mamshz})_{8}(\mathrm{DMA})_{6}\left(\mathrm{H}_{2} \mathrm{O}\right)_{2}\right] \cdot 2 \mathrm{DMA} \cdot 4 \mathrm{H}_{2} \mathrm{O}$ (1) were obtained by slow diffusion of manganese(II) acetate into a DMA (N,N-dimethylacetamide) solution of $\mathrm{H}_{3}$ mamshz at room temperature over a period of 4 weeks $\left(\mathrm{H}_{3}\right.$ mamshz: $\left.\mathrm{Mn}(\mathrm{OAC})_{2} \cdot 4 \mathrm{H}_{2} \mathrm{O}=1: 1\right)$. Yield: $71 \%$. Melting point: $>300{ }^{\circ} \mathrm{C}$ (dec.). Elemental data for $\mathrm{C}_{256} \mathrm{H}_{332} \mathrm{Mn}_{16} \mathrm{~N}_{48} \mathrm{O}_{70}$ calc: $\mathrm{C}$ 50.57, H 5.50, N 11.06\%; found: C 50.43, H 5.64, N 11.21\%. IR (KBr pellet, $\mathrm{cm}^{-1}$ ): 3433(br), 2922(m), 1608(s), 1562(s), 1498(s), 1427(s), 1394(s), 1350(s), 1327(s), 1246(s), 1215(m), 1176(s), 1161(w), 1119(m), 1061(w), 1018(m), 960(s), 928(s), 877(m), 816(m), 779(w), 764(s), 748(s), 698(m), 679(s), 648(s), 626(s), 594(m), 563(m), 513(m), 476(m), 442(s). 


\section{RESULTS AND DISCUSSION}

\section{IR spectra}

The IR spectra of $\mathrm{H}_{3}$ mamshz and compound $\mathbf{1}$ are listed in Table 1. The data of compound $\mathbf{1}$ show the stretching vibration of the $\mathrm{C}=\mathrm{O}$ band shifted to lower frequencies and the $\mathrm{O}-\mathrm{H}$ (phenolic) shifted to higher frequencies compared with the ligand, suggesting the coordination of carbonyl oxygen atom and the $\mathrm{O}$ (phenolic) atom to $\mathrm{Mn}^{3+}$ ion. The stretching vibration band $(\mathrm{N}-\mathrm{H})$ disappeared in the spectra of compound 1 indicating the deprotonation of the group and coordination of the hydrazide nitrogen atom to the $\mathrm{Mn}^{3+}$ ion. The bands of $\mathrm{C}-\mathrm{N}$ at 1286 and $1315 \mathrm{~cm}^{-1}$ are shifted to $1562 \mathrm{~cm}^{-1}$ due to the deprotonation of $\mathrm{NH}$ group.

Table 1. IR spectra of $\mathrm{H}_{3}$ mamshz and compound $\mathbf{1}$.

\begin{tabular}{|c|c|c|c|c|}
\hline Compound & $v(\mathrm{C}=\mathrm{O})$ & $v(\mathrm{O}-\mathrm{H})$ (phenolic) & $v(\mathrm{~N}-\mathrm{H})$ & $\delta(\mathrm{C}-\mathrm{H})$ (aromatic) \\
\hline $\mathrm{H}_{3}$ mamshz & 1660 & 3320 & 3040 & 769 \\
\hline $\mathbf{1}$ & 1610 & 3440 & - & 681 \\
\hline
\end{tabular}

Table 2. Crystal data and structure refinement of compound $\mathbf{1}$.

\begin{tabular}{|c|c|}
\hline Identification code & Compound $\mathbf{1}$ \\
\hline $\mathrm{CCDC}$ & 796822 \\
\hline Empirical formula & $\mathrm{C}_{25} 6 \mathrm{H}_{332} \mathrm{~N}_{48} \mathrm{O}_{70} \mathrm{Mn}_{16}$ \\
\hline Formula weight & 6077.4 \\
\hline Temperature $(\mathrm{K})$ & $296(2)$ \\
\hline Wavelength $(\AA)$ & 0.71073 \\
\hline Crystal system & Triclinic \\
\hline Space group & $P_{E_{1} 1} 1$ \\
\hline$a(\AA)$ & $20.725(5)$ \\
\hline$b(\AA)$ & $26.712(6)$ \\
\hline$c(\AA)$ & $29.357(7)$ \\
\hline$\alpha / \mathrm{deg}$ & $77.353(4)$ \\
\hline$\beta / \mathrm{deg}$ & $73.717(3)$ \\
\hline$\gamma / \mathrm{deg}$ & $74.031(3)$ \\
\hline Volume $\left(\AA^{3}\right)$ & $14820(6)$ \\
\hline$Z$ & 2 \\
\hline Calculated density $\left(\mathrm{Mg} \mathrm{m}^{-3}\right)$ & 1.360 \\
\hline Absorption coefficient $\left(\mathrm{mm}^{-1}\right)$ & 0.738 \\
\hline $\mathrm{F}(000)$ & 3174 \\
\hline Crystal size $\left(\mathrm{mm}^{3}\right)$ & $0.310 \times 0.267 \times 0.225$ \\
\hline Range for data collection & $0.994^{\circ}$ to $25^{\circ}$ \\
\hline Independent reflections & 19645 \\
\hline Refinement method & Full-matrix least-squares on $F^{2}$ \\
\hline Limiting indices & $-19 \leq h \leq 22,-28 \leq k \leq 28,-31 \leq l \leq 31$ \\
\hline Goodness-of-fit on $F^{2}$ & 1.030 \\
\hline Final $\mathrm{R}$ indices $[I>2 \operatorname{sigma}(\mathrm{I})]$ & $\mathrm{R}^{1}=0.0962, \mathrm{R}^{2}=0.2655$ \\
\hline Largest diff. peak and hole $\left(\mathrm{eA}^{-3}\right)$ & 1.539 and -0.998 \\
\hline
\end{tabular}

\section{$X$-ray diffraction data of compound 1}

The molecular structure of the title azametallacrown is shown in Figure 1. Single-crystal X-ray analysis showed that compound $\mathbf{1}$ crystallizes in triclinic space group $P-1$. There are two octanuclear metallamacrocycle: $\left[\mathrm{Mn}_{8}(\mathrm{mamshz})_{8}(\mathrm{DMA})_{8}\right]$ (ring A) and 
$\left[\mathrm{Mn}_{8}(\text { mamshz })_{8}(\mathrm{DMA})_{6}\left(\mathrm{H}_{2} \mathrm{O}\right)_{2}\right]$ (ring B), two DMA and four $\mathrm{H}_{2} \mathrm{O}$ molecules in an asymmetric unit (Table 1). Eight $\mathrm{Mn}$ (III) ions and eight deprotonated $N$-methacryl-5-methylsalicylhydrazidate $\left(\right.$ mamshz $\left.^{3-}\right)$ ligands assemble to form a planar 24-membered ring based on Mn-N-N-Mn linkage in the title azametallacrown. The ligand binds to the metal in a back-toback fashion, resulting in an octanuclear cyclic structure. The adoption of a sequence of chiralities by a certain kind of macrocycle is triggered by the tendency of the system to choose the lowest energy macrocycle (Figure 1, Table 2).

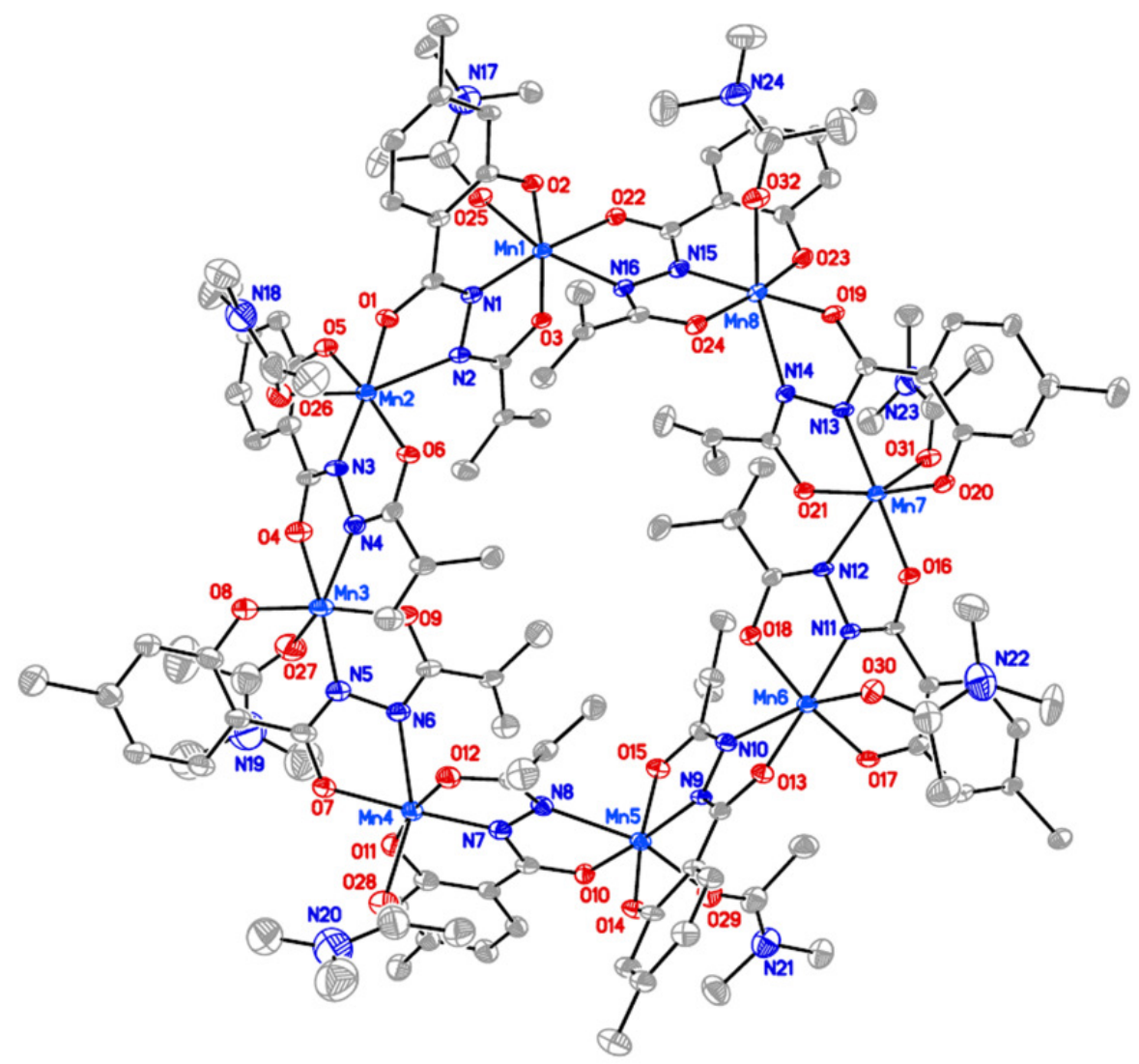

Figure 1. Molecular structure of the ring A of the title compound $\mathbf{1}$ with $\mathbf{M n}, \mathrm{N}, \mathrm{O}$ atoms labeling. $\mathrm{H}$ atoms and free solvent molecules are omitted for clarity.

All manganese ions in $\mathbf{1}$ are in a distorted octahedral $\mathrm{MnN}_{2} \mathrm{O}_{4}$ environment with a very distinct Jahn-Teller extension. The resulting octanuclear systems measure $\sim 21.5 \AA$ in diameter and $\sim 10 \AA$ in thickness. The neighboring Mn...Mn interatomic distances are 4.904(2)-4.983(2) $\AA$ and 4.871(2)-4.984(2) § in ring A and ring B, respectively.

The $\mathrm{Mn} . . . \mathrm{Mn} . . . \mathrm{Mn}$ interatomic angles in the 24-membered core rings are in the range 121.07(3)-134.38(2) ${ }^{\circ}$ and 121.32(3)-130.27(4) ${ }^{\circ}$ in the ring A and ring B, respectively. These values are quite close to the value of the interior angle in an $n$-octagon $\left(135^{\circ}\right)$. The bond distances for $\mathrm{Mn}-\mathrm{N}$ (diazine), Mn-O (phenolate), and Mn-O (carbonyl) are in the ranges 1.92- 
2.36, 1.85-1.89, and 1.94-1.99 ^, respectively. The Mn(III)-O(DMA) bond distances are from 2.21 to $2.30 \AA$. The $\mathrm{Mn}(\mathrm{III})-\mathrm{O}\left(\mathrm{H}_{2} \mathrm{O}\right)$ bond distances are from 2.24 to $2.25 \AA$. The terminal groups of the tails of 4 ligands are directed to the top of the cyclic structure, while the rest are directed down the cycle. The disc like molecule offers a cavity with an entrance diameter $~ 3.6$ $\AA$ and $\sim 12.9 \AA$ at the interior which may serve as potential molecular recognition site. There are no molecules in the 'host' cavity of the metallacrown.

The local environment of each metal centre of high-spin $d^{4} \mathrm{Mn}(\mathrm{III})$ ion has the same JahnTeller elongated octahedral geometry as in other manganese metallamacrocycles [6]. The JahnTeller distortion results in elongation of the axial manganese-oxygen/nitrogen bond lengths by $0.3 \AA$ relative to the other $\mathrm{Mn}-\mathrm{O} / \mathrm{N}$ bond lengths. The cyclic structure of the compound leaves a nano disc-shaped molecule (Table 3, Table 4).

Table 3. Selected bond lengths (Å) of compound $\mathbf{1}$.

\begin{tabular}{|c|c|c|c|c|c|c|c|}
\hline Bond & Length & Bond & Length & Bond & Length & Bond & Length \\
\hline Mn1-O2 & $1.858(6)$ & Mn3-O8 & $1.874(9)$ & Mn5-O14 & $1.861(8)$ & Mn7-O20 & $1.852(7)$ \\
\hline Mn1-O3 & $1.899(6)$ & Mn3-O9 & $1.945(8)$ & Mn5-O15 & $1.918(7)$ & Mn7-O21 & $1.934(7)$ \\
\hline Mn1-N1 & $1.939(8)$ & Mn3-N5 & $1.960(9)$ & Mn5-O10 & $1.943(7)$ & Mn7-N13 & $1.961(8)$ \\
\hline Mn1-O22 & $1.951(6)$ & Mn3-O4 & $1.995(8)$ & Mn5-N9 & $1.947(8)$ & Mn7-O16 & $1.979(6)$ \\
\hline Mn1-N16 & $2.271(8)$ & Mn3-O27 & $2.212(11)$ & Mn5-O29 & $2.207(9)$ & Mn7-O31 & $2.286(8)$ \\
\hline Mn1-O25 & $2.302(7)$ & Mn3-N4 & $2.261(9)$ & Mn5-N8 & $2.297(9)$ & Mn7-N12 & $2.319(8)$ \\
\hline Mn2-O5 & $1.854(7)$ & Mn4-O11 & $1.873(8)$ & Mn6-O17 & $1.865(7)$ & Mn8-O23 & $1.862(7)$ \\
\hline Mn2-N3 & $1.925(8)$ & $\mathrm{Mn} 4-O 12$ & $1.941(8)$ & Mn6-O18 & $1.931(7)$ & Mn8-O24 & $1.927(7)$ \\
\hline Mn2-O6 & $1.929(7)$ & $\mathrm{Mn} 4-\mathrm{N} 7$ & $1.949(9)$ & Mn6-N11 & $1.940(8)$ & Mn8-N5 & $1.945(8)$ \\
\hline Mn2-O1 & $1.952(7)$ & Mn4-O7 & $1.980(8)$ & Mn6-O13 & $1.955(7)$ & Mn8-O19 & $1.950(7)$ \\
\hline Mn2-O26 & $2.276(8)$ & Mn4-O28 & $2.237(9)$ & Mn6-O30 & $2.303(7)$ & Mn8-O32 & $2.288(8)$ \\
\hline Mn2-N2 & $2.358(8)$ & Mn4-N6 & $2.265(9)$ & Mn6-N10 & $2.349(9)$ & Mn8-N14 & $2.345(8)$ \\
\hline
\end{tabular}

Table 4. Selected bond angles of Compound 1.

\begin{tabular}{|c|c|c|c|c|c|}
\hline Angles & $\left(^{\circ}\right)$ & Angles & $\left(^{\circ}\right)$ & Angles & $\left(^{\circ}\right)$ \\
\hline O2-Mn1-O3 & $172.1(3)$ & N1-Mn1-O22 & $172.7(3)$ & O2-Mn1-O25 & $86.7(3)$ \\
\hline O2-Mn1-N1 & $92.3(3)$ & O2-Mn1-N16 & $89.7(3)$ & O3-Mn1-O25 & $92.2(3)$ \\
\hline O3-Mn1-N1 & $79.9(3)$ & O3-Mn1-N16 & $93.2(3)$ & N1-Mn1-O25 & $91.2(3)$ \\
\hline O2-Mn1-O22 & $94.5(3)$ & N1-Mn1-N16 & $102.1(3)$ & O22-Mn1-O25 & $91.9(3)$ \\
\hline O3-Mn1-O22 & $93.4(3)$ & O22-Mn1-N16 & $75.2(3)$ & N16-Mn1-O25 & $166.3(3)$ \\
\hline
\end{tabular}

\section{Magnetic measurements}

The temperature dependence of the magnetic susceptibility of compound $\mathbf{1}$ has been determined at a 1000 Oe field in 2-300 $\mathrm{K}$ temperature range. The diamagnetic correction was evaluated using Pascal's constants. As shown in Figure 2, the observed $\chi_{\mathrm{M}} T$ value for Compound $\mathbf{1}$ at 300 $\mathrm{K}$ is $58.67 \mathrm{~cm}^{3} \mathrm{~K} \mathrm{~mol}^{-1}$ and upon cooling from 300 to $35 \mathrm{~K}, \chi_{\mathrm{M}} T$ decreases slowly to approximately $30.24 \mathrm{~cm}^{3} \mathrm{~K} \mathrm{~mol}^{-1}$ and then drops very rapidly to reach $2.41 \mathrm{~cm}^{3} \mathrm{~K} \mathrm{~mol}^{-1}$ at $2 \mathrm{~K}$. Figure 2 shows a typical weakly coupled antiferromagnetic behaviour. Thus, by fitting the magnetic susceptibility data at high temperatures, $\mathrm{T}>35 \mathrm{~K}$, to the Curie Weiss expression $\chi^{-1}(\mathrm{~T})$ $=\mathrm{C} /(\mathrm{T}+\Theta)$, we obtained the Weiss constant, $\Theta=-32.61 \mathrm{~K}$ for $\mathbf{1}$. 


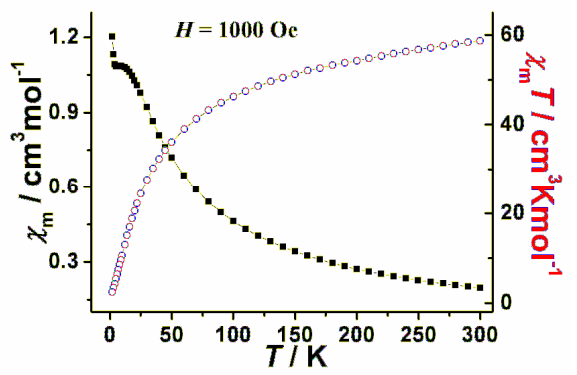

Figure 2. Plot of $\chi_{\mathrm{M}} T$ and $\chi^{-1}$ vs. T of polycrystalline sample of $\mathbf{1}$.

\section{CONCLUSION}

In this study, we were able to prepare a novel metallamacrocycle $\left[\mathrm{Mn}_{8}\left(\mathrm{mamshz}_{8}(\mathrm{DMA})_{8}\right]\left[\mathrm{Mn}_{8}(\mathrm{mamshz})_{8}(\mathrm{DMA})_{6}\left(\mathrm{H}_{2} \mathrm{O}\right)_{2}\right] \cdot 2 \mathrm{DMA} \cdot 4 \mathrm{H}_{2} \mathrm{O}\right.$. The systematic and detailed investigation of the factors that govern the sequence of the stereochemistry of the metal center, the nuclearity and size of the macrocycle, and the final architecture of this type of macrocyclic assembly are currently underway.

The supplementary crystallographic data for (1) have been deposited under the number CCDC 796822 and can be obtained free of charge from The Cambridge Crystallographic Data Centre via www.ccdc.cam.ac.uk/data_request/cif.

\section{ACKNOWLEDGEMENTS}

This work was supported by the Education Department of Shaanxi Province as a special research project (09JK512) and the Talent Fund of Ningbo Municipal Natural Science Foundation (No. 2010A610187).

\section{REFERENCES}

1. John, R.P.; Moon, D.; Lah, M.S. Supra. Chem. 2007, 19, 295; Loi, M.; Hosseini, M.W.; Jouaiti, A.; Cian A.D.; Fischer, J. Eur. J. Inorg. Chem. 1999, 1981; Xiao, F. P.; Jin, L.F.; Luo, W.; Cheng, G.Z.; Ji, Z. Inorg. Chim. Acta 2007, 360, 3341.

2. Mezei, G.; Baran, P.; Raptis, R.G. Angew. Chem. Int. Ed. 2004, 43, 574; Ni, Z.H.; Zhang, L.F.; Tangoulis, V.; Wernsdorfer, W.; Cui, A.L.; Sato, O.; Kou, H.Z. Inorg. Chem. 2007, 46, 6029.

3. Pereira, C.L.M.; Pedroso, E.F.; Stumpf, H.O.; Novak, M.A.; Richard, L.; Garcia, R.R.; Riviere, E.; Journaux, Y. Angew. Chem. Int. Ed. 2004, 43, 956; Brechin, E.K.; Cador, O.; Caneschi, A.; Cadiou, C.; Harris, S.G.; Parsons, S.; Vonci, M.; Winpenny, R.E.P. Chem. Commun. 2002, 1860.

4. Moon, M.; Kim, I.; Lah, M.S. Inorg. Chem. 2000, 39, 2710.

5. Su, C.Y.; Cai, Y.P.; Chen, C.L.; Smith, M.D.; Kaim, W.; Loye, H.C. J. Am. Chem. Soc. 2003, 125, 8595.

6. Kwak, B.; Rhee, H.; Park, S.; Lah, M.S. Polyhedron 2000, 19, 1985; Kim, I.; Kwak, B.; Lah, M.S. Inorg. Chim. Acta 2001, 17, 12; Lin, S.; Liu, S.X.; Huang, J.Q.; Lin, C.C. J. Chem. Soc., Dalton Trans. 2002, 1595.

7. Lin, S.; Liu, S.X.; Chen, Z.; Lin, B.Zh.; Gao, S. Inorg. Chem. 2004, 43, 2222. 